\title{
Simulations for CALET Energy Calibration Confirmed Using CERN-SPS Beam Tests
}

\author{
Yosui Akaike* for the CALET Collaboration \\ Waseda University, 17 Kikuicho Shinjuku-ku, Tokyo, Japan \\ E-mail: yosui.akaikedaoni.waseda.jp
}

\begin{abstract}
The CALorimetric Electron Telescope (CALET) is a detector for the precise measurement of cosmic ray electrons, gamma-rays, and nuclei on the International Space Station. CALET has an imaging and a thick calorimeter, which provide excellent energy resolution and particle identification. For the on-orbit calibration, it is uses the minimum ionizing particles of cosmic rays such as protons and Helium nuclei. We have carried out Monte Carlo simulations to develop an algorithm of penetrating event selection by event reconstruction and to estimate the on-orbit event rate for the calibration. We have also carried out the beam tests at the CERN-SPS to assess the detector performance and the validity of our Monte Carlo simulation and calibration methods. In this paper, we present the calibration methods and expected detector performance with beam test results.
\end{abstract}

The 34th International Cosmic Ray Conference,

30 July- 6 August, 2015

The Hague, The Netherlands

* Speaker. 


\section{Introduction}

The CALorimetric Electron Telescope (CALET) will be installed on the International Space Station (ISS) for precise measurements of $1 \mathrm{GeV}-20 \mathrm{TeV}$ electrons, $4 \mathrm{GeV}-10 \mathrm{TeV}$ gammarays and several $10 \mathrm{GeV}-1000 \mathrm{TeV}$ nuclei[四]. The goal of the CALET mission is to reveal unresolved high energy phenomena in the Universe. Recently, to explain the excess of electrons and positrons[ם, 目], several possible interpretations, such as pair creation of electrons and positrons in nearby astrophysical sources like pulsars or annihilation/decay of dark matter, have been suggested by numerous papers. Therefore, the high precision measurements of electron spectrum at $\mathrm{TeV}$ region is necessary to limit theoretical models by the search for a sharp cutoff and/or fine spectral structures. Although the measurement of line gamma-rays as dark matter signatures is also useful to limit the model, high energy resolution is required for the detector. Besides, the fact that the power indices of nuclei such as protons and Helium become harder above $\sim 100 \mathrm{GeV} / \mathrm{n}$ was reported by PAMELA[四] and AMS-02[回].

The measurements of wider energy range beyond the $\mathrm{TeV} / \mathrm{n}$ region with high energy resolution is required for the detailed study on the hardening of the spectra. CALET has a capability to achieve excellent energy resolution, $\sim 2 \%(>100 \mathrm{GeV})$ for electrons and gamma-rays, and particle identification, $\sim 10^{5}$ proton rejection power, from $\mathrm{GeV}$ to $\mathrm{TeV}$ regions with its imaging and thick calorimeter, which has been estimated by Monte Carlo simulations[ [G, प]. However, in order to realize such an excellent performance in observation, a careful calibration is necessary. The detector response of each component including the light yield of scintillator, the gain of PMT/APD, the noise and so on, can be totally calibrated by using minimum ionizing particles (MIP). We have proved the expected performance in beam tests for electrons and protons, and balloon experiments for electrons and atmospheric gamma-rays by the calibration using muons [ $\mathbb{Q}, \mathbf{Q}]$. The ground calibration of flight instruments using cosmic ray muons has been carried out. On-orbit calibration will be carried out, considering the environmental condition, thermal, radiation, etc. in space, using penetrating cosmic ray events. In case of space observation, cosmic ray protons and Helium nuclei are useful for the calibration. Because CALET has a thickness of $1.3 \lambda$ for protons, about $30 \%$ of protons can penetrate without interactions through the whole detector in vertical direction.

The beam test at CERN-SPS in 2015 have been carried out to evaluate the detector performance for heavy nuclei using the fragments of Argon, and to access our Monte Carlo simulations. In this beam test, we calibrated the detector by selecting the penetrating events of Helium and verified the performance of the measurement of shower events as preliminary results. We have also carried out Monte Carlo simulations to develop an algorithm to select penetrating events and to estimate the event rate to be used for calibration. In this paper, we present expected detector performance with the beam test results and the calibration methods. We mainly focus on the calibration of the PWO logs because it directly affects the energy spectrum we will obtain from CALET observation. According to the simulations, nearly $90 \%$ of the initial energy of $1 \mathrm{TeV}$ electrons is deposited in the TASC, and we can get energy resolution less than $3 \%$ just using the TASC in the energy region over $100 \mathrm{GeV}[$ [6]. 


\section{CALET Calorimeter}

The CALET detector is composed of Charge Detector (CHD), Imaging Calorimeter (IMC), and Total Absorption Calorimeter (TASC). CHD is used for charge identification of nuclei. It is composed of two orthogonal layers of 14 scintillator paddles, each of which has dimensions of 3.2 $\mathrm{cm} \times 44.5 \mathrm{~cm} \times 1.0 \mathrm{~cm}$. IMC is adopted to detect the pre-shower image and to identify the charge, the shower start point and the arrival direction of incident particles. This part is composed of 7 tungsten plates and 8 detection layers made of scintillating fiber (SciFi). Each layer consists of two SciFi belts arranged in the $x$ and $y$ direction. Each SciFi belt is assembled of 448 SciFis of $1 \mathrm{~mm}$ square cross section and $448 \mathrm{~mm}$ length. TASC is located at the bottom for precise measurement of both energy and identification of the incident particle. TASC is composed of 12 layers arranged alternately in the $x$ and $y$ direction as a hodoscope. Each layer has 16 PWO logs; each log has dimensions $1.9 \mathrm{~cm} \times 32.6 \mathrm{~cm} \times 2.0 \mathrm{~cm}$. An avalanche photodiode (APD) - photodiode (PD) pair is attached to each PWO log to achieve a measurement with very wide dynamic range more than $\left(10^{6}\right)[\square]$.

\section{CERN-SPS Beam Test}

We carried out a beam test in 2015 at CERN-SPS using fragments of Argon with energies of 13, 19 and $150 \mathrm{AGeV/c}$. As a detector, we used the CALET structural thermal model, which has the same geometry as the flight model. The details are described in reference [[3].

In order to calibrate the detector components, we used the single penetrating events of Helium, applying the selection methods as follows. In case of the beam test, since all events are incident vertically to the detector, the rejection of side escaping or entering events is not necessary. Besides, since all events have enough energy to pass through the detector, the rejection of stopping events in the detector is not necessary as well. Therefore the event selection is a bit easier than that of

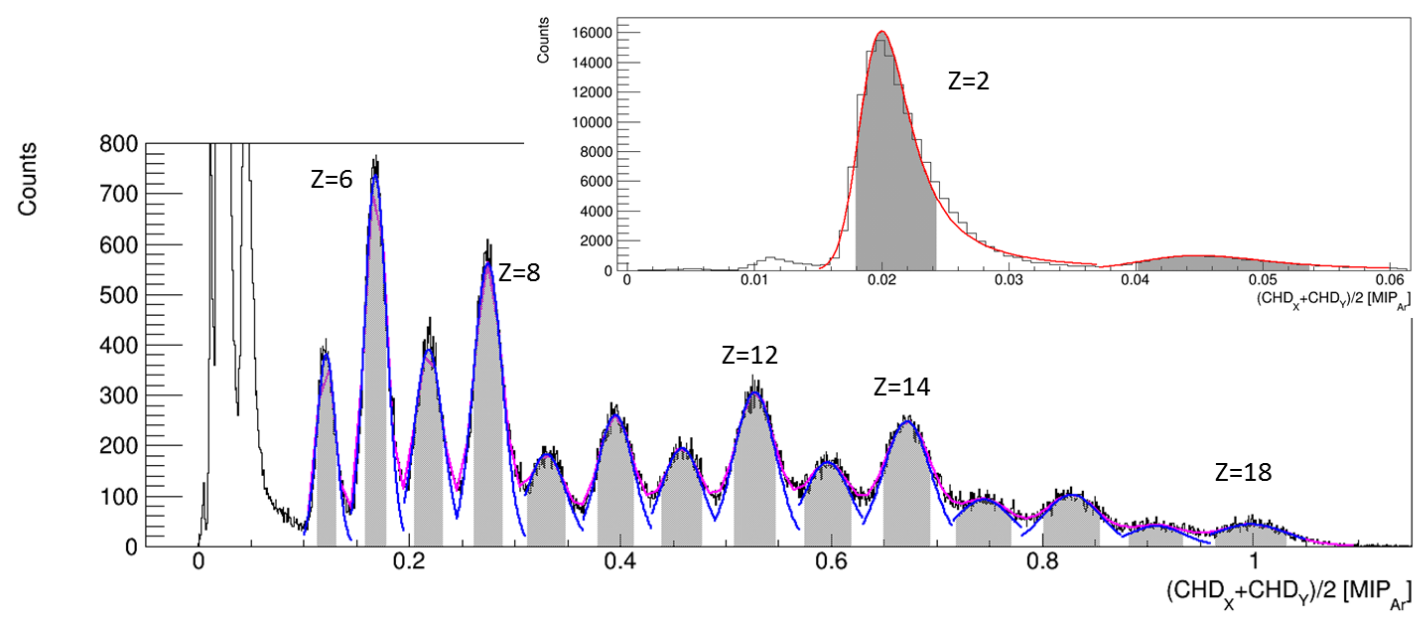

Figure 1: The number of particles in CHD paddles. The gray area shows the 1 sigma region of each element; these events are used to analyze the MIP particles and shower measurements. 

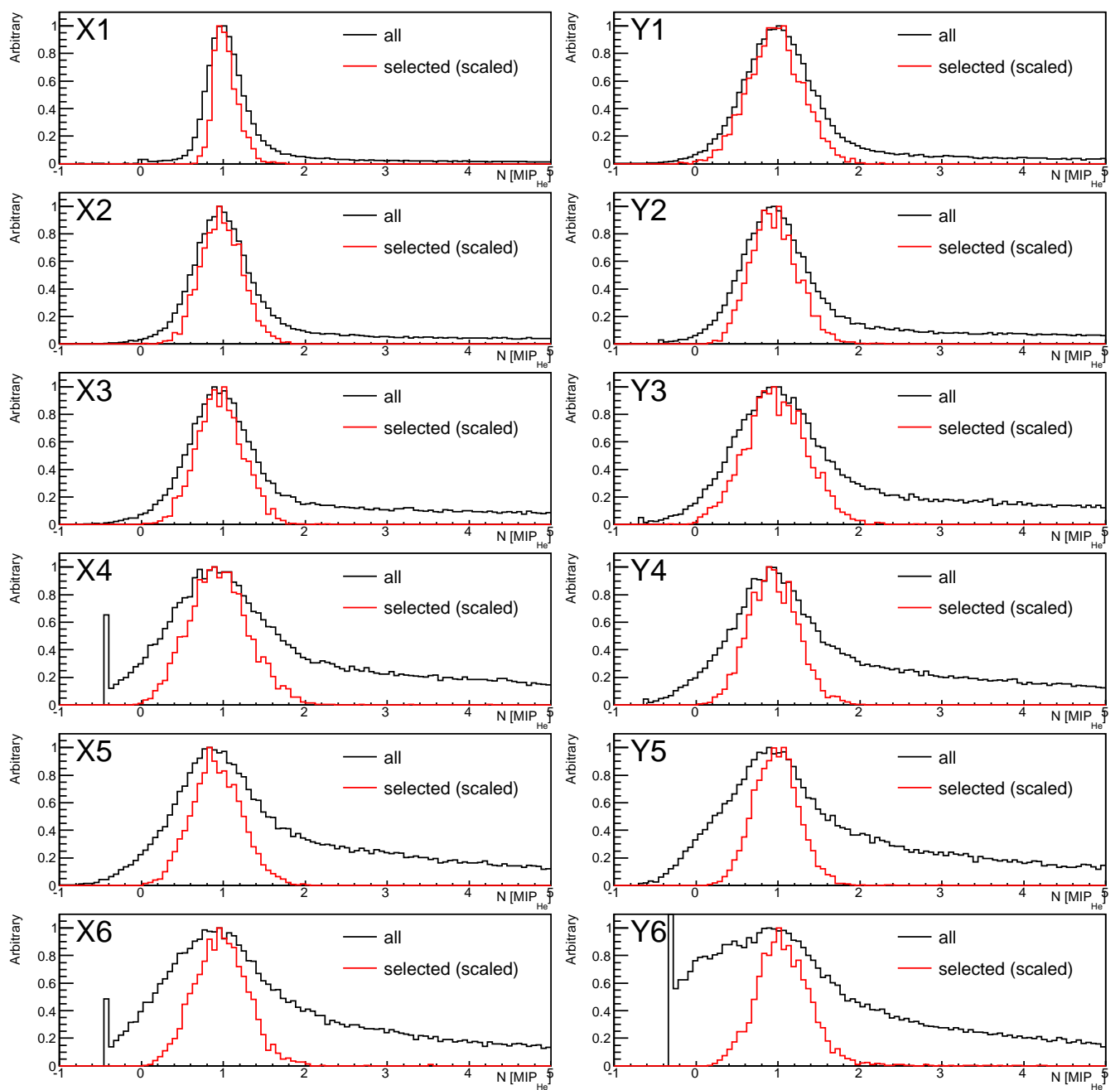

Figure 2: Distributions of signals of one PWO log in each layer. The black lines represent the distribution before the event selection, and the red lines represent the distribution after the event selection.

on-orbit calibration. However, the rejection of shower events are still important. According to the simulation, since $86 \%$ of $13 \mathrm{AGeV} / \mathrm{c}$ Helium nuclei interact in the detector and create shower particles, we rejected these events. And then, we have evaluated the performance of the shower energy measurements compared with Monte Carlo simulations. We used the EPICS code[[प]] with DPMJET-III[L5] as a hadron interaction model.

\subsection{Selection of Penetrating Helium Nuclei}

At first, in order to select hit CHD paddles and hit PWO logs, the incident direction is reconstructed using IMC data. The track reconstruction was done by least-squares fit of the most luminous fibers in each IMC layer. Particles from the accelerator included all fragments with $A / Z=2.0$. We selected the Helium events using the CHD signals. Figure $\mathbb{W}$ shows distributions of the average number of particles in CHD-X and Y paddles. Each channel of the CHD was normalized at $Z=18$ for the pure Ar beam. Charges of $Z<4$ are fitted by a Landau function 

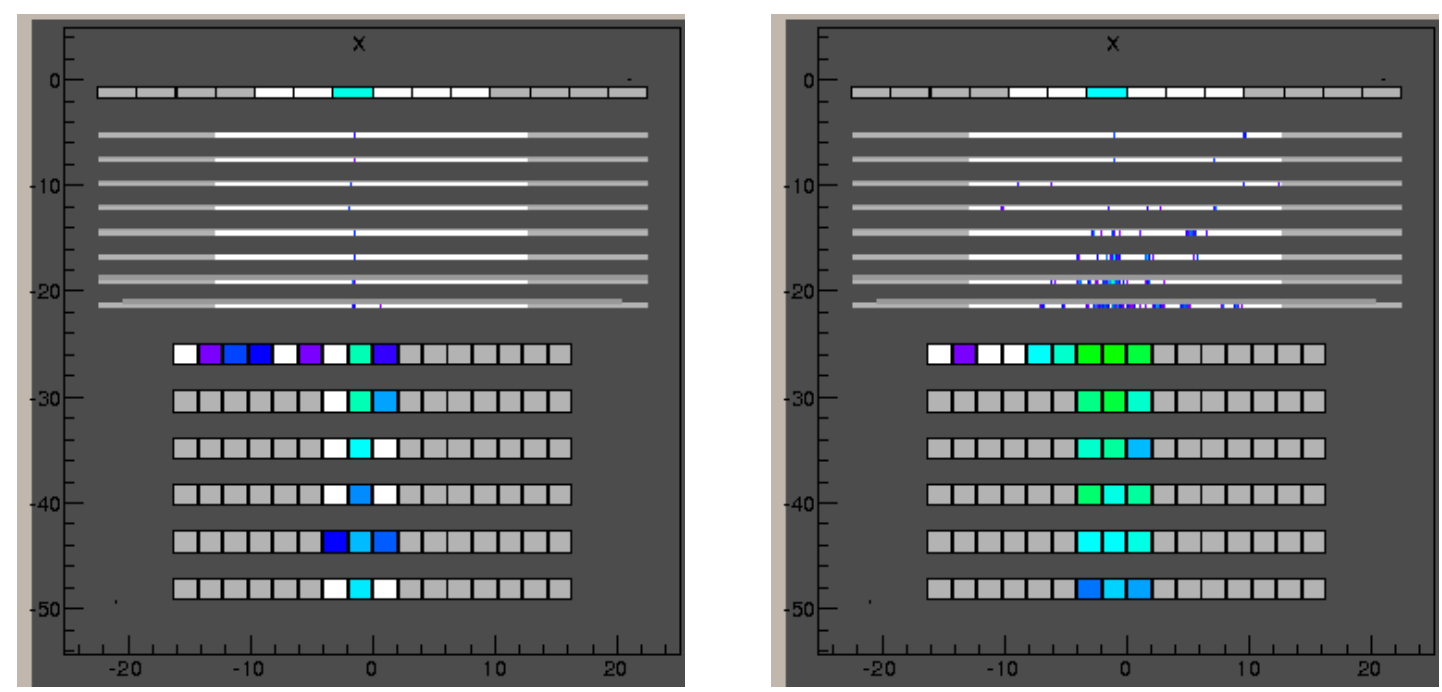

Figure 3: Examples of $\mathrm{X}$-side detection image of $\mathrm{He} 13 \mathrm{AGeV} / \mathrm{c}$ beam. Left: a penetrating event, right: a shower event interacted at IMC. Color contour shows the scale of energy deposition.

convoluted with a Gaussian, and charges of $Z>4$ are fitted by a Gaussian. In the following analysis, we used the events within the one sigma region around the peak for each element.

To select the penetrating events, we use a "single-likeness" parameter. Using a template distribution $p(x)$ of penetrating events based on Monte Carlo simulations including the r.m.s. noise of pedestal for each channel respectively, the likelihood of single events can be estimated for each event as $P=\Pi_{i} p\left(x_{i}\right)$ where $x_{i}$ is the signal in the $i$ th layer. Figure $\square$ shows the distributions of the number of particles at the center PWO log in each TASC layer. The black lines show the distributions for all events whose reconstructed tracks in the IMC pass through the PWO log, and the red lines show the events selected by the likelihood analysis. It can be seen that the shower events in the right-hand tails are effectively removed. Events on the left side of the peaks in the figures (black lines) are mis-reconstructed tracks and tracks affected by elastic scattering; these are also effectively removed. Figure 13 shows examples of events selected as a penetrating event and a shower event that interacting in the IMC.

\subsection{Shower Energy Compared with MC}

We used APD high gain and low gain channels which are calibrated by the correlation plots between APD high gain and low gain [Q]. As an example, the distributions of the total number of particles in TASC derived from $150 \mathrm{AGeV} / \mathrm{c}$ Silicon test beam (TB) events tagged by the CHD are

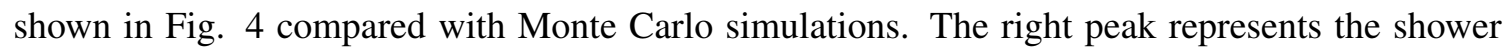
events and left peak represents the penetrating events. The penetrating event peak in the Monte Carlo simulation is larger than that of the test beam data, because we have not applied the quenching effect into the PWO signals in this simulation. The quenching effect will be included in future analysis.

In order to evaluate the performance of shower energy measurement with MIP calibration, we selected the shower events that interacted in the IMC using the information of the bottom IMC layers. This approach to identify interacting events in the IMC is still preliminary and independent 


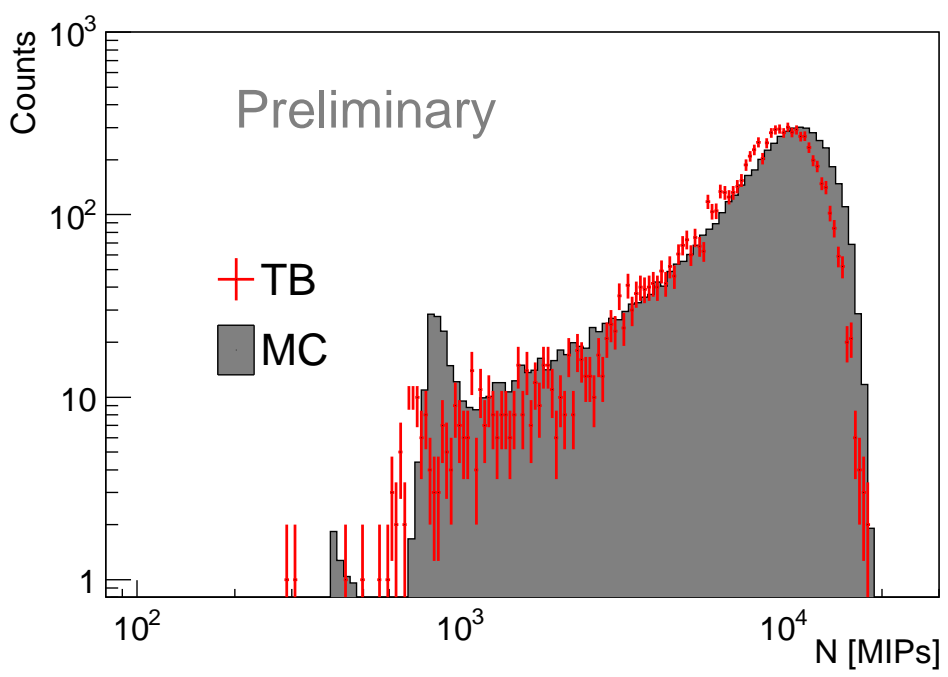

Figure 4: The distribution of the number of particles (150 AGeV/c Silicon) compared with Monte Carlo simulations.

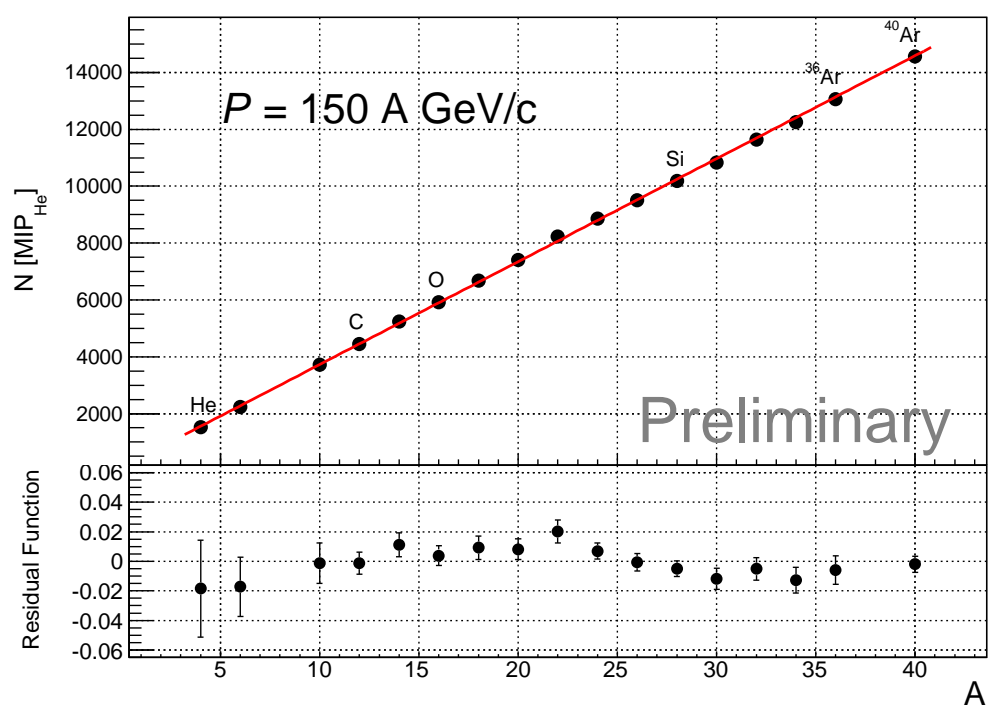

Figure 5: The TASC signal, in units of number of $\mathrm{MIP}_{\mathrm{He}}$, which is a measure of shower energy, as a function of the mass number for $150 \mathrm{AGeV} / \mathrm{c}^{40} \mathrm{Ar}$ primary beam and the fragments with $A / Z=2.0$.

of that in reference[ए]]. Figure $[$ shows the linearity of the measured shower energy as a function of incident mass number for $150 \mathrm{AGeV} / \mathrm{c}$ Helium to Argon. In this analysis, we used ${ }^{40} \mathrm{Ar}$ primary beam and fragment beam selected with $A / Z=2.0$. Although the result is preliminary, we can confirm that the residual value is less than $2 \%$ up to $6 \mathrm{TeV}$.

\section{Energy Calibration on the ISS Orbit}

We have been carrying out Monte Carlo simulations to develop an algorithm to select penetrating events which pass through the detector without creating showers and to estimate the 
event rate. Details are described in the reference [ए2], so we present here just an outline.

\subsection{Event Rate}

CALET has a specific trigger mode for calibration to detect penetrating events. According to our calculation of the flux of protons and Helium nuclei at the ISS orbit, the estimated trigger rate with the single trigger is $\sim 140 \mathrm{~Hz}$ on average, resulting in $6.3 \times 10^{5}$ protons and $1.3 \times 10^{5}$ Helium nucleus triggers during one orbital period (90 minutes). For the detector calibration we should select events which pass through the detector from the top to the bottom without escaping or entering through the side, nor stopping inside the detector, nor creating shower particles. $4.1 \%$ of the triggered protons and $1.8 \%$ of the triggered Helium nuclei are expected to satisfy these conditions, and the number of useful events during one orbital period is $2.6 \times 10^{4}$ for protons and $2.3 \times 10^{3}$ for Helium nuclei.

\subsection{Event Selection On-Orbit}

When we use protons and Helium nuclei for the detector calibration, we need a careful analysis to separate penetrating particles from shower events. The first step of the event selection is a track reconstruction using IMC data. This reconstruction allows the identification of the hit PWO for almost $77 \%$ of single penetrating protons. The other events are affected by reconstruction errors of elastic scattering in the detector. As the second step of the selection, we check the total deposited energy in the TASC. Shower events make a large energy deposition in the TASC so more than $70 \%$ of them can easily be cut. As the third step, we use a likelihood analysis using a "single-likeness" parameter as described in previous section. $90 \%$ of shower events and $95 \%$ of stopping events can be rejected by this cut. The purity of the selected events in the final selection step is more than $76 \%$, while more than $67 \%$ of single penetrating events are retained. These values have a dependence of geomagnetic latitude because of the different rigidity cutoff.

\subsection{Calibration Accuracy}

The signal distribution after the event selection can be fitted with a Landau function convoluted with a Gaussian. The error of the most probable value of the Landau function expected in one orbital period is less than $3 \%$. The error becomes smaller with higher statistics (for example, $1.0 \%$ in 5 orbital periods).

\section{Conclusions}

We have carried out beam tests at CERN-SPS to verify the detector performance and test our Monte Carlo simulations. The electron and proton tests allowed us to calibrate the detector using muons and obtain the performance expected by simulations. The heavy ion beam tests allowed us to use the penetrating Helium events to evaluate the performance. We verified the applicability of the calibration methods using Helium nuclei and obtained good linearity of the energy measurements up to $6 \mathrm{TeV}$. The full analysis of the beam test results continues and the results presented here is still preliminary.

We also carried out Monte Carlo simulations of the performance expected on the ISS orbit using cosmic ray protons and Helium nuclei. The expected calibration error of each channel with 
the statistics of one ISS orbit, which is a reasonable amount of time, is less than 3\%. This error is small enough to maintain the performance such as the high energy resolution, $\sim 2 \%$.

\section{Acknowledgement}

We sincerely thank CERN-SPS beam coordinators. This work was supported by JSPS KAKENHI Grant Numbers 15K17636 and 25257203.

\section{References}

[1] S. Torii et al., Calorimetric Electron Telescope (CALET) for the International Space Station, in this proceedings of the 34th International Cosmic Ray Conference

[2] O. Adriani et al., An anomalous positron abundance in cosmic rays with energies $1.5-100 \mathrm{GeV}$, Nature 458 (2009) 607

[3] L. Accardo et al., High Statistics Measurement of the Positron Fraction in Primary Cosmic Rays of 0.5 - $500 \mathrm{GeV}$ with the Alpha Magnetic Spectrometer on the International Space Station, PRL 113 (2014) 121101

[4] O. Adriani et al., PAMELA Measurements of Cosmic-Ray Proton and Helium Spectra, Science 332 (2011) 6025

[5] M. Aguilar et al., Precision Measurement of the Proton Flux in Primary Cosmic Rays from Rigidity 1 GV to 1.8 TV with the Alpha Magnetic Spectrometer on the International Space Station PRL 114 (2015) 171103

[6] Y. Akaike et al., Expected CALET Telescope Performance from Monte Carlo Simulations Proc. of 32nd ICRC, (2011) 769

[7] F. Palma et al., Simulation Studies of the Expected Proton Rejection Capabilities of CALET, in this proceedings of the 34th International Cosmic Ray Conference

[8] Y. Akaike et al., CALET Observational Performance Expected by CERN Beam Test Proc. of 33rd ICRC, (2013) 726

[9] G. Bigongiari et al., CALET Perspectives for Calorimetric Measurements of High Energy Electrons Based on Beam Test Results, in this proceedings of the 34th International Cosmic Ray Conference

[10] T. Niita et al., A Balloon Experiment Using CALET Prototype (bCALET-2), Adv. Space Res. 55 (2015) 753

[11] D. Ito et al., High-dynamic Range Readout System Using Dual APD/PD for the CALET-TASC, Proc. of 33rd ICRC, (2011) 824

[12] T. Niita et al., Energy Calibration of Calorimetric Electron Telescope (CALET) in Space, Adv. Space Res. 55 (2015) 2500

[13] T. Tamura et al., Heavy Ion Beam Test at CERN-SPS with the CALET Structure Thermal Model, in this proceedings of the 34th International Cosmic Ray Conference

[14] K. Kasahara et al., Introduction to cosmos and some relevance to ultra high energy cosmic ray air showers, Proc. of 24th ICRC (1995)

[15] S. Roesler et al., The Monte Carlo Event Generator DPMJET-III, Proc. Monte Carlo 71 (2000) 23

[16] P. Brogi et al., CALET measurements with cosmic nuclei: expected performances of tracking and charge identification, in this proceedings of the 34th International Cosmic Ray Conference 